\section{A Silicon Multi-Cathode Detector For Microanalysis Applications}

Shaul Barkan and Liangyuan Feng

Radiant Detector Technologies, LLC

Jan S. Iwanczyk, Bradley E. Patt and Carolyn R. Tull

Photon Imaging, Inc.

Dale E. Newbury and John A. Small

National Institute of Standards \& Technology

\section{Introduction}

A new class of silicon multi-cathode detector ( $S M C D$ ) has been developed for microanalysis spectrometry applications. The detector has excellent energy resolution ( $<150 \mathrm{eV}$ FWHM) and high count rate capability ( $>1 \mathrm{Mcps}$ ). An energy resolution of $143 \mathrm{eV}$ FWHM at $5.9 \mathrm{keV}$ was measured with the SMCD at $6 \mu$ s peaking time. At a

\section{Spectrometer Package}

The elimination of cryogenic cooling components allows for the development of compact, low power electronics and a compact spectrometer package. The compact package also allows for more flexible measurement setups and adaptation to a large number of measurement environments.

A spectrometer containing a $0.5 \mathrm{~cm}^{2} S M C D$ detector, cooled using a small Peltier cooler, was custom-designed to fit within a scanning electron microscope (SEM). The spectrometer was installed on a JEOL 840 SEM at the National Institute of Standards and Technology (NIST). Figures 1 and 2 show photographs of the microanalysis version of the SMCD spectrometer package, as it was designed specifically for insertion into the SEM column. A very low noise FET (field effect transistor) and preamplifier are the first two readout stages for the SMCD. A digital pulse processor, optimized for very short peaking times and high count rate capability, was used for amplification and pulse-processing. Data collection and spectrum analysis was performed using a multi-channel analyzer with software designed specifically for the SMCD.

\section{Measurements on the JEOL SEM}

Figure 3 shows a copper spectrum collected from the SMCD on the SEM, at $15 \mathrm{KV}$ beam energy. The copper $L / K$ ratio is $8: 1$, which is indicative of a very thin dead layer in the entrance window of the silicon detector itself. On the same SEM, under similar conditions, the copper $\mathrm{L} / \mathrm{K}$ ratio measured with a conventional windowless $\mathrm{Si}(\mathrm{Li})$ detector was $5: 1$, indicating an increased transmission of the very low energy $x$-rays from the SMCD window.

The SEM was configured for $x$-ray mapping of an $\mathrm{AINi}(\mathrm{Fe})$ catalyst sample. Figure 4 shows the $x$-ray spectrum collected from this sample with the SMCD spectrom-

Figure 1. Photograph of SMCD spectrometer for microanalysis, which fits into the SEM column.

Figure 2. SMCD installed into JEOL 840 SEM at NIST.

very short peaking time of $250 \mathrm{~ns}$, an energy resolution of less than $240 \mathrm{eV}$ is easily achieved, with a commensurate output count rate of greater than $400 \mathrm{kcps}$. The new SMCD technology will be able to replace many existing detectors based on cryogenically cooled Si $(\mathrm{Li})$ and high purity germanium. The new SMCD represents a distinct advantage for $\mathrm{x}$-ray mapping, as the detector can be operated at very high count rates (without the typical unacceptable degradation in energy resolution), which then expedites the mapping collection time by an order of magnitude compared with existing techniques. Silicon Multi-Cathode Detector (SMCD)

The new SMCD is based on the silicon drift detector technology [1-3]. The multi-cathode detector design allows for the creation of a large active area detector $\left(0.5 \mathrm{~cm}^{2}\right.$ or greater $)$, but without the corresponding large capacitance that is presented by traditional pin $n$ diodes of similar area. The SMCD detectors have an extremely small capacitance $(-60 \mathrm{fF})$, which results in a very low series noise component and thus the corresponding capability to operate at short amplifier peaking times, while still maintaining excellent energy resolution. Operation at short peaking times then results in a high count rate capability.

The SMCD detector is fabricated on $0.35 \mathrm{~mm}$ thick, high resistivity float zone silicon, using conventional silicon CMOS (complimentary metal oxide silicon) processing techniques. The SMCD contains a large number of cathode rings surrounding a small signal collection anode. The cathode rings are precisely biased to create an electric field within the detector that causes all of the charge carriers within the detector volume to be collected in the very small anode region, resulting in excellent spectral quality. The detector face opposite the cathode ring side is a very thin window for optimum transmission of the low energy $x$-rays. In addition, the SMCD is thin enough to be cooled with only a thermoelectric cooler and thus does not require liquid nitrogen cooling. eter, while Figure 5 shows the corresponding compositional maps derived from the $x$-ray spectra.

\section{Conclusions}

Radiant Detector Technologies' new silicon multi-cathode detector (SMCD) shows great promise for $\mathrm{x}$-ray microanalysis applications

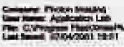

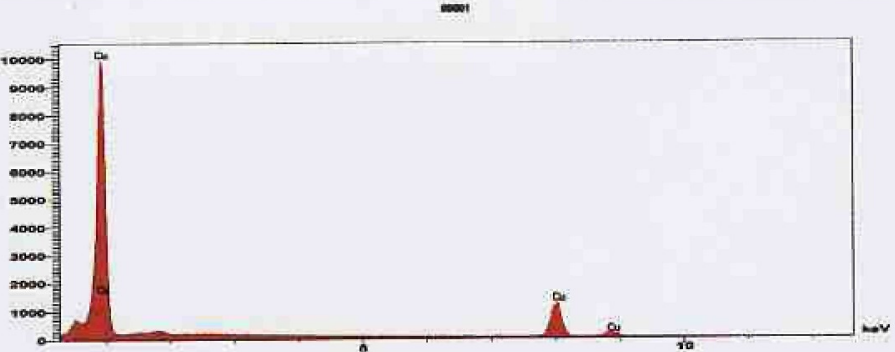

Figure 3. Characteristic $x$-rays of Cu measured with the SMCD spectrometer on the SEM.

\section{Exis}

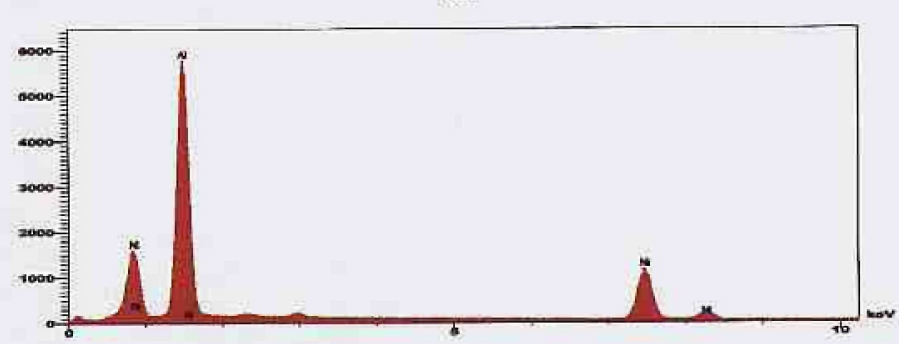

Figure 4. X-ray spectrum of an AINi(Fe) catalyst sample used for compositional mapping. 


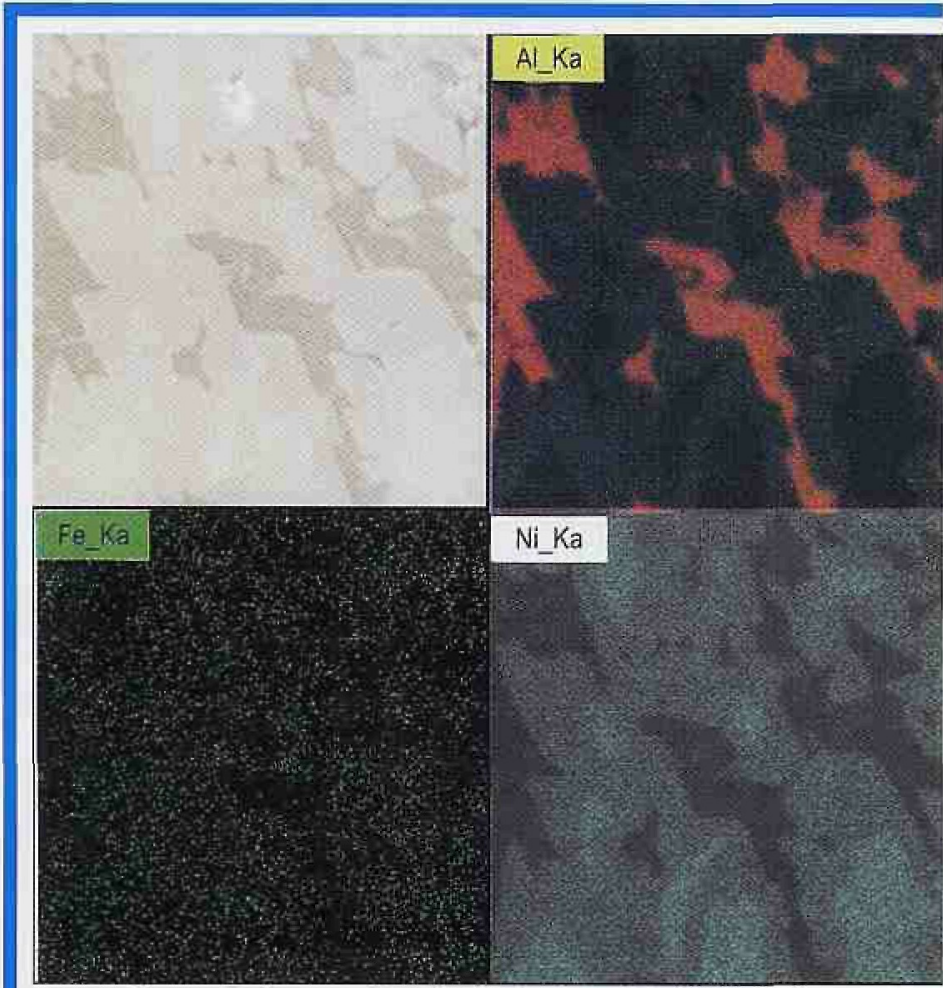

Figure 5. Compositional maps of an AINi(Fe) catalyst (130 $\mathrm{mm}$ field of view; $256 \times 256$ pixels; $50 \mathrm{~ms}$ dwell time)

and offers the following advantages over existing $x$-ray detector technologies:

a. Large active area $\left(\sim 0.5 \mathrm{~cm}^{2}\right)$

b. Elimination of cryogenic cooling requirements

c. Near room temperature operation with Peltier cooling, which enables flexible implementation of various detector configurations

d. Excellent energy resolution at short shaping times and high count rates

e. Very high count rate capability (1 Mcps), which is a special advantage for fast $x$-ray mapping

f. High sensitivity to low energy $x$-rays

\section{References}

1. Iwanczyk, B.E. Patt, C.R. Tull, J. Segal, C. Kenney, J. Bradiey, B. Hedman and K.O. Hodgson, "Large area silicon drift detectors for $x$-rays - new results", IEEE Trans. Nucl. Sci, vol. 46 (1999) 284-288.

2. S. Barkan, J.S. Iwanczyk, B.E. Patt, L. Feng, C.R. Tull and G. Vilkelis, "Vortex ${ }^{T M}$ - A new high performance silicon drift detector for XRD and XRF Applications", presented at the Denver X-Ray Conference, Aug. 2002, Denver $\mathrm{CO}$, to be published in Advances in X-Ray Analysis Vol 46 (2003).

3. J.S. Iwanczyk, B.E. Patt, L. Feng, S. Barkan, C.R. Tull; "High Throughput, High Resolution Silicon Drift Detectors for X-Rays", Presented at the IEEF Nucl. Sci. and Med, Imaging Symposium, Norfolk VA Nov, 2002; submitted to IEEE Trans. Nucl. Sci. Vol. 50 (2003)
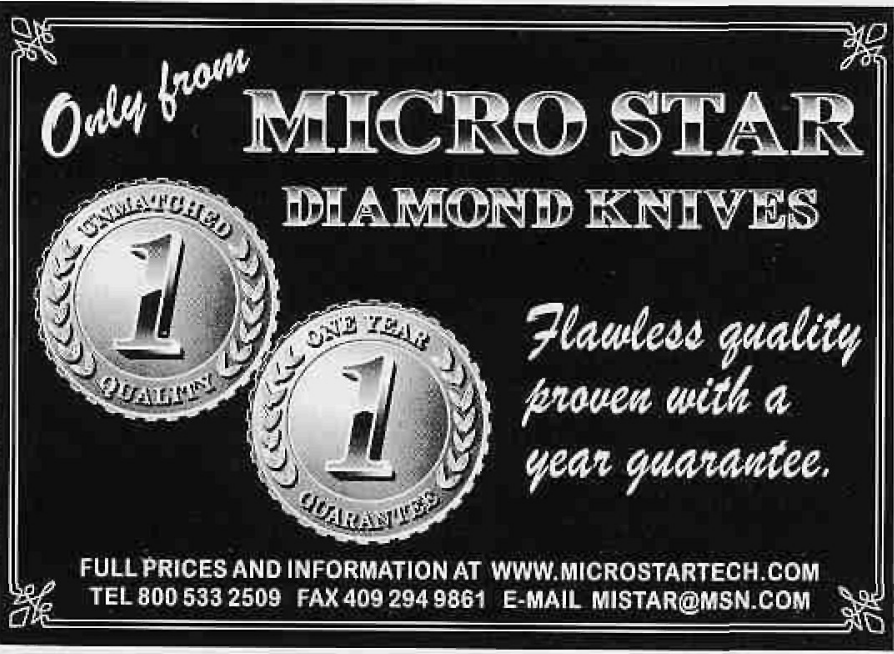

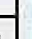

西
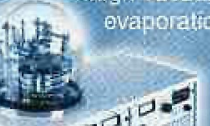

10.7

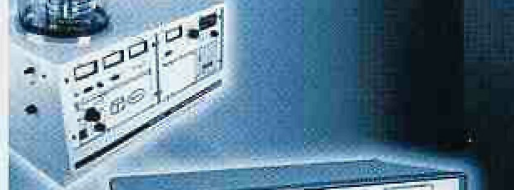

Advanced Preparation

Electron Mitrossopy

preparafion proiludis

from "POLARON"

the most respected name

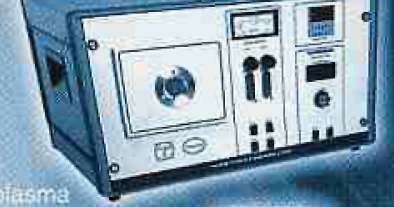

in the indusiry

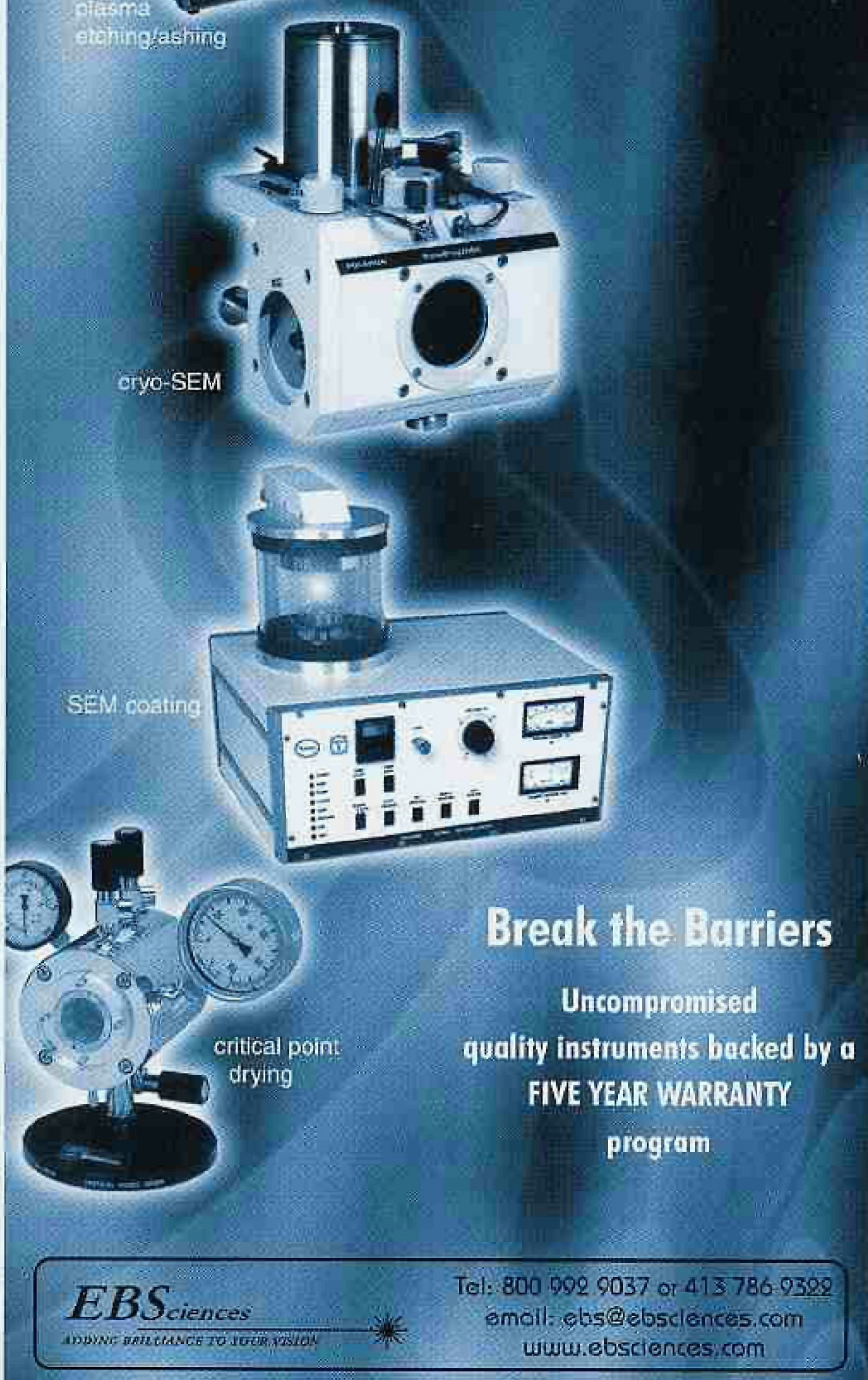

Contact us for critical point dryers, sputter couters, cartion evaporators;

high vacuum evoporators, plosmo reactors and many more.

\section{Wwa.quorumiech.com}

